\title{
Marquis de Sade, l'Empire et l'empire des métaphores : des Maudits aux quills et les autres plumes
}

\author{
Maja Vukušić Zorica \\ Faculté de Philosophie et Lettres \\ Université de Zagreb \\ mzorica@ffzg.hr
}

Le titre, délibérément ambivalent, annonce que l'analyse va procéder à deux temps : en premier lieu, partir du lien entre les dernières années de la vie ${ }^{1}$ de Marquis de Sade, qui meurt à Charenton le 2 décembre 1814, et la période de l'Empire. Dans une société marquée par la censure, il établit paradoxalement, au sein d'une maison de fou, un empire, celui de la liberté des passions sous l'égide du théâtre écrit, créé et mis en scène. De ce rapport entre l'asile et le théâtre, qui sous-entend le " pouvoir psychiatrique » foucauldien, dans cet empire des passions, naît une certaine scène, dans le sens ranciérien du terme, scène de la métaphorisation contemporaine.

A l'effondrement du modèle de la théâtralité spécifique du roman libertin correspond ici l'interprétation de Sade de l'écrivain croate Ivo Brešan dans son roman Prokletnici (Les Maudits), à travers la métaphore de la société croate en transition, et celle, américaine, de la pièce de théâtre de Doug Wright, Quills (Plumes) et sa métaphore de l'écriture. Le bilan est vacillant.

Mots-clés : Marquis de Sade, Empire, asile, théâtre, scène, roman libertin, Brešan, Wright

\section{Marquis et 1'Empire}

«C'est cela le sublime - mais au sens du XVIII e siècle c'est-à-dire la Terreur. On peut fonder historiquement la sublimation. C'est ainsi que Buguy a écrit en l'an V : 'La Révolution s'allégorisant pour faire oublier la Terreur, tel est l'Empire. » (Pascal Quignard, L'enfant d'Ingolstadt, Dernier royaume X, Paris, Bernard Grasset, $2018: 248)$

1 Le titre éponyme du tome 25 de la correspondance de Marquis de Sade, qui couvre la période de 1798 à 1814. Voir Alice M. Laborde, Correspondance du Marquis de Sade et de ses proches enrichies de documents notes et commentaires, Genève, Éditions Slatkine, 1997. 
Les dernières années de Sade couvrent la période de 1798 à 1814. En dehors de la période révolutionnaire, quand Sade jouit de sa liberté en devenant l'un des membres cruciaux de la Section des Piques, tout comme Robespierre, cette période est notamment la période de sa dernière incarcération.

Le 6 mars 1801 (15 ventôse an IX), Sade est arrêté avec l'imprimeur-libraire Massé $^{2}$ dans la maison de ce dernier, car en juillet ou en août 1800, Massé a publié en quatre volumes in- $12^{\circ}$ les Crimes de l'Amour (ou Les Contes), 453 feuillets représentant les petits récits multithématiques écrits, en partie, en même temps que le rouleau des 120 Journées de Sodome, pendant son séjour à la Bastille, au cours de l'année 1785.

Les Crimes de l'amour font scandale ; en témoigne l'article de Victor-Donation de Musset-Pathay (1768-1832), père d'Alfred de Musset, dans la Décade philosophique ${ }^{3}$, qui fait voir que Sade est dans le collimateur, surveillé de près par les censeurs et les critiques depuis le Consulat, et certainement sous l'Empire.

Chez Massé la police trouve un exemplaire de la Nouvelle Justine avec les annotations de Sade et un exemplaire du dernier tome de L'Histoire de Juliette, et des manuscrits autographes (dont le Boccace Français et les Délassements du Libertin). Sade nie son statut d'auteur de Juliette et prétend n'être que le copiste (7 mars 1801), jusqu'au moment où Massé révèle où se trouvent cachés les 1000 exemplaires de Juliette (A.N., F ${ }^{18}, 1730$, \# 23 in Laborde 1997 : 231).

Après la saisie de ses manuscrits ${ }^{4}$ chez Massé, Sade est emprisonné à SaintePélagie $^{5}$ puis à Bicêtre, et transféré à l'hospice de Charenton-Saint-Maurice en

2 Journal typographique et bibliographique, ou recueil consacré à tout ce qui paraît de nouveau en Littérature, Sciences et Arts, $\mathrm{n}^{\circ}$ XLIII, 8 août 1800 [20 thermidor an VIII], p. 351, in Vial $2014: 60$.

3 « Fidèle aux principes qu'il a établis dans Aline et Valcour, D. A. F. Sade continue d'attribuer à l'amour presque tous les crimes qui déshonorent l'espèce humaine. On (je ne dis pas il) l'avait déjà fait dans un ouvrage qu'il était destiné à notre siècle de produire : ouvrage infâme dont on n'ose même répéter le titre, tout décent qu'il paraît. L'Auteur des Crimes de l'Amour a fait une dénégation solennelle de cette infernale production. Malheureusement dans tous ses ouvrages, il est question et toujours question des crimes de l'amour; dans ceux qu'il nie, comme dans ceux qu'il avoue, ce sont toujours les crimes de l'amour ! Voilà sans doute ce qui a fait attribuer le premier à l'auteur des autres. Quand on voit les mêmes principes, la même morale, le même style, on a quelque droit de porter ce jugement. Je plains bien sincèrement D. A. F. Sade d'être dans le cas de se justifier » (La Décade philosophique, littéraire et politique, n 9, 30 frimaire an IX [21 décembre 1800], p. 542 in Viala 2014:66)

4 La disparition du troisième manuscrit de la préfecture de police qu'évoque Vial (2014:64) a été attribuée au chef de division à la préfecture de police, Jean-Baptiste Boucheseiche (1760-1825), responsable de la surveillance de Sade devant le préfet de police et le ministre Fouché, qui présida à l'arrestation et à l'internement de Sade le 6 mars 1801 et qui le surveilla jusqu'à la mort du marquis. Le feuillet sauvé (collection d'Henri de Rothschild), le seul connu du manuscrit de l'Histoire de Juliette, publiée dès 1799, est en effet la page de titre. Ainsi Juliette lui vaut-il un nouveau internement.

5 Le 2 avril 1801 (12 germinal an IX), Sade est incarcéré à Sainte-Pélagie (Laborde 1997 : 233-234). Le préfet Dubois au ministre de la Police, 26 mai 1801, 6 prairial an IX (A.N., $\mathrm{F}^{7} 7294$, \#30, in Laborde 1997 : 234-235). 
avril $1803^{6}$, où il passera les 11 dernières années de sa vie en « semi-liberté », et où il meurt le 2 décembre $1814^{7}$ à l'âge de 74 ans.

Charenton est un hospice (et non une prison) dirigé par François Simonet de Coulmier jusqu'à sa démission en mai 1814, quand il va être remplacé par un avocat, Roulhac de Maupas, le 7 septembre 1814, trois mois avant la mort de Sade. Or, le docteur Gastaldi/Gastaldy, que Sade connaît depuis des années, va jouer un rôle déterminant sur le sort de Sade, car il partageait ses idées nouvelles sur les soins et le comportement envers les malades, notamment les effets bénéfiques du théâtre. Le 22 décembre 1805, avec la mort de son ami Gastaldi/y, la situation change radicalement : Gastaldy est remplacé par le docteur Antoine-Anasthase RoyerCollard (1768-1825) qui n'apprécie pas cette nouvelle méthode, et veut supprimer la permission donnée à Sade de diriger les activités théâtrales (Laborde 1997: 251) ${ }^{8}$.

Après plusieurs années d'intervention obstinée auprès des autorités politiques, Royer-Collard réussit à faire supprimer les spectacles de Charenton par un arrêté ministriél le 6 mai 1813 (Laborde 1997 : 280)ำ.

En 1814, ses manuscrits circulent et se revendent ${ }^{10}$ et même après sa mort, ils sont problématiques : par exemple, la « Note relative aux manuscrits de M. de Sade, Paris, 17 décembre 1814, (A.N., F7 6294, \# 5 » in Laborde 1997 : 394) vise

6 En mars 1803, Sade est écroué à la prison de Bicêtre, en mi-avril (25 germinal an XI), et le préfet de Police, Dubois, effectue le transfert à l'hospice de Charenton-Saint-Maurice le 27 avril 1803 que sa famille avait obtenu de la police (Laborde 1997 : 240).

7 Dans la note de Charenton, après le 31 mars 1813 «(B.N., MS. 24390, fo. 414) (Laborde 1997 : 371), Sade confirme avoir été interrogé trois fois à Charenton au cours de sa dernière incarcération (le 31 mars 1811 par le Comte Jolivet, le 14 novembre 1813 par le Comte Corviette (ou Dorvietto) et le 31 mars 1813, par le Comte Appellius ou un nom semblable)

8 «Le dimanche 14 avril 1805 (24 germinal, an XIII), Sade distribue le pain bénit et fait la quête en l'église paroissiale de Charenton-Saint-Maurice à l'occasion de la fête de Pâques. Trois jours plus tard le préfet de police Dubois adresse la réprimande et les ordres [...] à M. Coulmier » de la défense de « sortir sans une autorisation expresse et formelle de (s) a part »(Laborde 1997 : 251). Sade communiait chaque année depuis 1791, pour honorer sa libération de la prison où il était depuis 1777 (Ibid. : 252). La Police générale de Paris, « $3^{\mathrm{e}}$ arrondissement, 6029, Charenton, le 10 juin 1806, (A.N., F $\mathrm{F}^{7}$ 6294, ๑) 20 » rejette sa demande de permission de sortir deux fois par semaine (Laborde 1997 : 259). Le préfet le fait encore le 23 juillet 1808 car un commissaire de police a extrait Sade de l'hospice le 20 juillet pour aller chez un notaire, en prétendant que le préfet l'en a autorisé ( « Préfecture de Police, $2^{\mathrm{e}}$ Division, $3^{\mathrm{e}}$ Bureau, Paris, le 23 juillet 2808, (B.N., MS. 24390, ff. 291-292 » in Laborde 1997 : 276-277). Le Sénateur ministre le confirme à M. le Conseiller d'État préfet de Police (expédié le 20 août 1808, (A.N., F76294, ® 17 in Laborde 1997 : 277).

9 « M. de Sade à Mme de Santeuil, Charenton, juillet 1813, (J.L.D., 567-568)» (Laborde 1997 : 371). Elle est mentionnée en 1808, puis le 5 octobre 1814, quand elle lit le journal à Sade pour la première fois. Ici il la prie d'empêcher son mari, les deux surveillants à Charenton (elle se remariera avec le fils de Mme Quesnet), de ne pas signaler à M. de Coulmier de prétendus signaux qu'il se défend d'avoir fait à une femme.

10 M. Branzon les avait achetés de M. Laccord, Inspecteur au Service des vivres militaires («Extrait des registres de correspondance du secrétariat général de la Police de Paris, Paris, 18 mai 1814, (A.N., F 6294, ๑ 5) » in Laborde 1997: 373. 
à interdire de les faire imprimer à Londres, et de les remettre à la famille pour qu'elle les brûle ${ }^{11}$.

Selon Laborde (1997 : 229), ces dernières années semblent être « les plus calmes, les plus fructueuses et jusqu'à un certain point les plus heureuses de sa vie », même si, après la mort des Montreuil, de sa femme Renée-Pélagie, qui meurt en 1810, et des vilenies de ses deux fils, dont l'aîné meurt en 1809, il n'a que Madame Quesnet, sa compagne dévouée, de son côté.

Ainsi les dernières années de Sade font-elles voir trois thèmes cruciaux : la loi et son statut d'auteur qu'il nie, la folie et le théâtre.

\section{La loi ${ }^{12}$ :}

Sade cache souvent son statut d'auteur (La Philosophie dans le boudoir est proclamé « œuvre posthume » en 1795) ou le nie (il se dit copiste et non pas l'auteur de l'Histoire de Juliette en 1801) et en déconseille la lecture. Les Journées de Florbelle, achevées en prison, où Sade revendique son statut d'auteur, sont une exception (Vial 2014 : 66). Dans la correspondance, Sade affirme plusieurs fois ne pas être l'auteur de Justine :

«[...] Il circule dans Paris un ouvrage infâme ayant pour titre : Justine ou les malheurs de la vertu ; plus de deux ans auparavant, j'avais fait paraître un roman de moi intitulé : Aline et Valcour ou le roman philosophique, malheureusement pour moi, il a plu à l'exécrable auteur de Justine de me voler une situation, mais qu'il a obscénisée, luxuriorisée, de la plus dégoûtante manière ; il n'en a pas fallu davantage pour faire dire à mes ennemis que les deux ouvrages m’appartenaient et que puisque j'avouais Aline, c'était clair que Justine sortait également de ma plume.

Pendant ce temps-là, on me tendit des pièges, on m'envoya de jolies femmes à Saint-Ouen (mais inconnues de moi) qui me priaient à mains jointes de leur céder un exemplaire de Justine. [...] SADE ${ }^{13}$ » (« Le citoyen Sade à

11 «L'inventaire après décès de M. le comte de Sade, 2 janvier 1815, 9 heures du matin, (B.N., MS. 24384, ff. 603-621) » in Laborde 1997 : 394-414) comprend la bibliothèque de son cabinet, ses papiers et manuscrits.

12 Il est surveillé par la police dont témoigne l'« État sommaire des pièces du citoyen Donatien Alphonse François Sade déposés dans les bureaux du ministre de la police générale », « 19 brumaire 1800, 28 brumaire an IX (A.N., $\mathrm{F}^{7} 4954^{3}$, \# 34 », depuis 1790) (Laborde 1997 : 219-224). En 1806, Sade écrit son Testament (Charenton-Saint-Maurice, 30 janvier 1806, (B.N., MS. 24384, ff. 599-603) qui comprend la cinquième clause, avec les indications précises au sujet du traitement de son corps après la mort (Laborde 1997 : 256-257). Le 16 janvier 1811 Sade va enfin, après des années, obtenir l'amnistie générale décidée par Savary, duc de Rovigo, ministre de la Police générale, concernant tous les faits d'émigration, et par la suite, le préfet de la Seine, Frochot, va lui accorder le 5 mars 1811 une main levée générale de séquestre (Laborde 1997 : 350).

13 Et encore, dans une lettre à un destinataire non identifié (Saint-Ouen, 7 septembre 1800, 20 fructidor, (J.L.D., 470-472), in Laborde 1997 : 216-217), probablement à M. Delaporte, « secrétaire de la Comédie-Française en 1791 », selon Laborde, quand Sade répond à 
un destinataire non identifié, Versailles, an VIII, 1800, (J.L.D., 467-468), in : Alice M. Laborde 1997 : 214)

Plusieurs fois il fait appel à la loi pour déterminer son statut ; il écrit au ministre Fouché, tout convaincu de son droit à la liberté (« Le citoyen Sade au citoyen Fouché (Joseph Fouché (1759-1820)), Pélagie, ce 20 mai 1802, 30 floréal an X, (copie reprise à « Revue Rétrospective », 1833, $1^{\text {er }}, 256$ ), Sade, homme de lettre au ministre de la Justice » (Laborde 1997 : 236-237) :

« Je veux, en un mot, être libre ou jugé. J'ai le droit de parler ainsi. Mes malheurs et les lois me le donnent et j'ai lieu de tout espérer quand c'est à vous que je m'adresse » $(1997: 237)$.

Dans sa lettre du 12 août 1804, il se plaint de son incarcération « depuis près de quatre ans »(«Le citoyen Sade au ministre Fouché / A Mgr Fouché, Ministre de la Police générale, Sade, homme de lettres, 12 août 1804, 24 thermidor, an XII (A.N., F.7 6294, \# 24) in Laborde 1997 : 247) :

«Les lois et règlements sur la liberté individuelle n'ont jamais été aussi ouvertement enfreints qu'en ce qui me concerne, puisque c'est sans jugement ni acte légal quelconque que l'on persiste à me retenir sous les verrous, soidisant en raison d'un ouvrage obscène qu'on m'attribue mal à propos et aussi en raison de quelques fables qu'on se plaît à imaginer et appliquer à ma vie privée, le tout sans fondement. En conséquence, Monseigneur, je me trouve dans le cas de recourir à votre autorité et surtout à votre équité, pour obtenir mon élargissement, puisque non seulement toutes les lois et la raison sont méconnues en ce qui me concerne, mais encore parce que l'un et l'autre commandent textuellement qu'on me rende libre. $[\ldots]^{14}$ »

la demande de son ouvrage, probablement Les Crimes de l'amour, que venait de publier Massé, qu'il assume, tout en niant d'être l'auteur de Justine : "Il est impossible que Madame Quesnet vous ait promis Justine, car, non seulement, elle n'en a aucune à sa disposition, mais bien convaincue que cet ouvrage n'est pas de moi, elle sait bien de même que je n'en ai aucune à la mienne ; croyez-moi, Monsieur, ne soyez point curieux de ce très mauvais libre, il fait frémir et si j'avais eu dans un moment de délire, le malheur de le créer, j'aurais assez de raison aujourd'hui pour couper la main qui l'aurait écrite. [...] SADE ». Et encore : «[...] Détenu depuis neuf mois à Pélagie comme prévenu d'avoir fait le livre de Justine, qui pourtant n'émana jamais de moi, je souffre et ne dis mot, comptant chaque jour sur la justice du gouvernement ; mais lorsque des méchants, désespérés de mon silence et de ma résignation, cherchent à me nuire par tous les moyens possibles, je les démasque. (Il se plaint d'un prisonnier qui lui a volé des poésies pour les faire imprimer et comme dans le volume, il y en a contre le premier Consul, il s'élève avec force contre cette publication et proteste de son attachement inviolable aux principes républicains) SADE » («Le citoyen Sade au Ministre de la Police, Pélagie, 26 décembre 1801, 5 nivôse an X, (Coll. A Bouër) » in Laborde 1997 : 235).

14 Il écrit une nouvelle fois au ministre Fouché (« Le citoyen Sade au ministre Fouché, N 136 du Bureau de la Liberté individuelle, A Monseigneur Fouché ministre de la police générale de l'empire français ; Sade homme de lettres. Charenton, 28 août 1804, 10 fructidor an XII, (A.N., F7 6294, \#21, in Laborde 1997 : 249-250), où il le demande de « lui accorder la permission d'aller à Paris pour vaquer à ses affaires, toutes les fois 
Le rapport de la « Police générale de l’Empire, Division de sûreté générale, RAPPORT, 18 août 1804, thermidor an XII, (A.N., F7 6294, \#23, \# 28 » (Laborde 1997 : 248) qui traite la réclamation de Sade laisse voir le caractère arbitraire de cette incarcération :

« Il paraît qu'il n'y aurait pas de preuve suffisante pour le faire juger. D'ailleurs la loi ne prononce pour ces délits qu'une détention de quelques mois et il ne paraîtrait pas convenable que cette condamnation fût prononcée après une détention de trois années. La longue détention qu'il a subie doit l'avoir corrigé. Son Excellence déterminera s'il ne serait pas à propos de prendre un parti à son égard».

Or, à la suite de ces lettres, le Conseiller d'État, préfet de police, refuse de le libérer et propose de le laisser à Charenton («Police générale, N6029, SADE, Paris, 8 septembre 1804, Du 21 fructidor an 12, (A.N., F 6294, \# 25 » in Laborde 1997 : 250).

En 1804, le préfet de Paris, Louis-Nicolas Dubois (1758-1847) écrit un rapport à Joseph Fouché, ministre de la police générale (1759-1820), pour répondre aux lettres assidues de Sade de Charenton au gouvernement impérial :

«Son Excellence le sénateur, ministre de la police générale de l’Empire, par sa note du 7 de ce mois me demande un rapport sur le nommé Sade, détenu à Charenton. Dans les premiers jours de ventôse an IX, j'avais été informé que le nommé Sade, ex-marquis, connu pour être l'auteur de l'infâme roman de Justine, se proposait de publier incessamment un ouvrage plus affreux encore sous le titre de Juliette. Je le fis arrêter le 15 du même mois chez le libraire éditeur de son ouvrage, où je savais qu'il devait se trouver muni de son manuscrit. L'auteur et l'éditeur furent amenés à ma préfecture. La saisie du manuscrit était importante ; mais l'ouvrage était imprimé, et il s'agissait de découvrir l'édition. La liberté fut promise à l'éditeur, s'il livrait les exemplaires imprimés. Celui-ci conduisit nos agents dans un lieu inhabité que lui seul connaissait, et ils en enlevèrent une quantité assez considérable d'exemplaires pour que l'on pût croire que c'était l'édition entière. Sade, dans son interrogatoire, reconnut son manuscrit, mais il déclara qu'il n'était que le copiste et non l'auteur. Il convint même qu'il avait été payé pour le copier, mais il ne put faire connaître les personnes de qui il tenait les originaux. Il eût été difficile de croire qu'un homme qui jouissait d'une fortune assez considérable eût pu devenir copiste d'ouvrages aussi affreux, moyennant un salaire. On ne pouvait douter qu'il n'en fût l'auteur, lui dont le cabinet était tapissé de grands tableaux représentant les principales obscénités du roman de Justine ${ }^{15}$ ».

que cela lui deviendra indispensable. Le régime de la maison de santé de Charenton où le suppliant a demandé d'être transféré et où il est maintenant s'arrange d'autant mieux à cette permission que cet hospice ne fut jamais regardé comme prison mais simplement comme maison de surveillance, spécialement pour ceux qui, dans le cas où Sade se trouve, n'en ont sollicité l'habitation que comme un adoucissement à la longueur de leur détention. »

15 Revue rétrospective ou bibliothèque historique, contenant des mémoires et documens authentiques, inédits et originaux..., Paris, H. Fournier aîné, 1833, t. I, p. 258-259, rapport du préfet de police Dubois à Fouché, ministre de la Police générale, Paris, 21 fructidor an XII [8 septembre 1804] in Vial 2014 : 66-67. 
Dubois écrit une nouvelle fois à Fouché, au sujet du manuscrit confisqué lors d'une des perquisitions à Charenton, le 5 juin $1807^{16}$, des Journées de Florbelle ou la Nature dévoilée, suivie des Mémoires de l'abbé de Madoz et des Aventures d'Émilie de Volnange ${ }^{17}$, commencés vers la fin des années $1790^{18}$ et mises au net en prison à partir du 5 mars 1806. Le manuscrit relégué dans «l'Enfer» des écrits subversifs / licencieux de la préfecture de police de Paris et détruit sous la Restauration par «M. Delavau, préfet de police, en présence et sur la demande de M. de Sade, le fils (Armand de Sade) », n'est sauvé partiellement (il ne survit que sous la forme de quelques feuillets photographiés par Maurice Heine) que grâce à la " curiosité » du secrétaire du préfet de police (Vial 2014 : 62). :

«[...] la lecture de cet ouvrage est révoltante. Il semble que Sade ait voulu aller au-delà des horreurs de Justine et de Juliette. On accumulait les épithètes les plus épouvantables que l'on ne caractériserait pas cette infernale production. Il est impossible de lire de suite ces dix volumes d'atrocités, de blasphèmes, de scélératesse. Il y règne constamment, au milieu de la débauche la plus raffinée, une extravagance raisonnée dont heureusement peu d'hommes sont capables » (François Ost, Sade et la loi, Paris, Odile Jacob, 2005, p. 64 in Vial $2014: 63)$.

Or, à l'encontre des accusations,les manuscrits licencieux semblent être les passe-temps favoris des après-dînées du préfet Dubois et de ses collègues du Conseil d'État, et de l'inspecteur général de la librairie, Veyrat, et ses amis ${ }^{19}$.

Le « Rapport sur la conduite de Sade à Sainte-Pélagie et à Bicêtre, Ministère de la Police générale, Secrétariat Général, Paris, le 21 avril 1809, (A.N., F76294, \# 8) » in Laborde 1997 : 287) fabrique des justificatifs après-coup : ce rapport l'accuse d'avoir séduit et corrompu les jeunes à Sainte-Pélagie, où « il voulut assouvir sa passion brutale sur de jeunes étourdis qui par suite de désordre commis au Théâtre Français avaient été envoyés quelques jours à Sainte-Pélagie » (Ibid.), d'où son transfert à Bicêtre et Charenton ${ }^{20}$.

16 Jean-Baptiste Jeangène Vilmer, Sade moraliste, Genève, Droz, 2005, p. 169 in Vial 2014 : 63.

17 Le titre définitif est adopté le 25 avril 1807, lors des dernières épreuves de l'ouvrage.

18 Une partie du manuscrit semble antérieure, datant de la fin des années 1790, comme le stipule Jean-Jacques Pauvert dans l'«Introduction » (CEuvres complètes du marquis de Sade. 11, Notes littéraires, couplets et pièces de circonstances; Notes pour les Journées de Florbelle, Paris, Pauvert, 1991, p. 60 in Vial 2014: 62).

19 Étienne-Denis Pasquier, Mémoires du chancelier Pasquier : histoire de mon temps, Paris, Plon-Nourrit et $C^{\text {ie }}$, 1893, vol. 1, p. 451, in Vial $2014: 63$ ).

20 «On trouva dans sa chambre un instrument énorme qu'il avait fabriqué avec de la cire et dont il s'était servi lui-même, car l'instrument avait conservé les traces de son introduction coupable. Cet homme étant dans un état perpétuel de démence libertine, fut transféré à Charenton dans la maison des fous, au mois de floréal de la même année. Il continua à y composer des romans plus affreux encore que Juliette. Et au mois de juin 1807, je fis saisir dans sa chambre plusieurs mémoires dont la lecture est révoltante. C'est une suite d'obscénités, de blasphèmes et de scélératesses qu'on ne 
En 1809, Sade écrit la seule lettre présente dans la correspondance adressée à Napoleon $1^{\mathrm{er}}$ :

«De Sade à Napoléon 1 ${ }^{\mathrm{er}}$, A Sa Majesté l'Empereur et Roi, Protecteur de la Confédération du Rhin en sa commission des pétitions au Conseil d'État, 17 juin 1809 (Dr. Cabanes, 312)

Sire,

Le sieur de Sade, père de famille dans le sein de laquelle il voit pour sa consolation un fils qui se distingue aux armées, traîne depuis plus de neuf ans, dans trois différentes prisons consécutives, la vie du monde la plus malheureuse.

Il est septuagénaire, presque aveugle, accablé de goutte et de rhumatismes dans la poitrine et dans l'estomac, qui lui font souffrir d'horribles douleurs, des certificats de médecins de la maison de Charenton, où il est maintenant, attestent la vérité de ces faits et l'autorisent à réclamer enfin sa liberté, en protestant qu'on n'aura jamais lieu de se repentir de la lui avoir donnée.

Il ose se dire, de Votre Majesté, Sire, avec le plus profond respect, le très humble, très obéissant serviteur et sujet de SADE » (Laborde 1997 : 289-290).

Napoléon a probablement vu Juliette, si ce n'est sous forme de manuscrit confisqué, il se rappelle, lors de son séjour à Sainte-Hélène, avoir parcouru « le livre le plus abominable qu'ait enfanté l'imagination la plus dépravée », dont le nom de l'auteur lui échappe ${ }^{21}$.

\section{La folie}

Dès 1810, les autorités veulent le transférer au Château d'If ou au Fort de Ham : «Minute du Ministre de l'Intérieur (Montalivet) relative au citoyen Sade, Paris, le 18 octobre 1810, (B.N., MS. 24390, fo. 10) » qui revendique de placer Sade « dans un local distinct et séparé de manière que toute communication soit dans l'intérieur soit à l'extérieur lui soit entièrement interdite » (Laborde 1997 : 329), suivie de la décision de «M. Montalivet, ministre de l'Intérieur à M. de Coulmier, directeur de l'Hospice de Charenton, Ministère de l'Intérieur, $3^{\mathrm{ème}}$ division, Bureau des secours, des hospices, des prisons et de la mendicité, Paris, le 18 octobre 1810, (B.N., MS. 24390, fo. 293) », qui le qualifie comme étant « atteint de la plus dangereuse de toutes les folies ; que ses communications avec les autres habitués de la Maison offrent des dangers incalculables, que ses écrits ne sont pas moins

peut caractériser. On y trouva également un instrument semblable à celui qu'on avait saisi dans sa chambre à Sainte-Pélagie » (Ibid.). Laborde (1997 : 288) explique que Sade avait été opéré au moins deux fois dans sa vie d'une fistule mal placée au niveau du sphincter anal, qui nécessita les soins constants, mettre de l'onguent avec un bâton de cire (le docteur Gastaldi assurait ces soins), d'où l'instrument en question.

21 Emmanuel de Las Cases, Mémorial de Sainte-Hélène, Paris, L'Auteur, 1823, t. VII, p. 283 in Vial 2014:67. 
insensés que ses paroles et sa conduite ; que ces dangers sont surtout imminents au milieu d'êtres dont l'imagination est déjà affaiblie ou égarée » (Ibid. : 329). Il revendique non seulement une réclusion totale, mais aussi l'interdiction de « tout usage de crayons, d'encre, de plumes et de papier » (Ibid. : 330).

«M. de Coulmier à son Exc. Mgr. Le Ministre de l'Intérieur, Charenton, ce 24 octobre 1810, (B.N., MS. 24390, ff. 11-12) » (Laborde 1997 : 330-332) déclare ne pas pouvoir lui donner un local isolé, mais que Sade « occupe dans ce moment la seule chambre dont toute communication à l'extérieur est interceptée », qu'il est le seul dépositaire de cette clef, et qu'il se verrait « humilié d'être un geôlier ».

Cette affirmation du directeur de l'hospice introduit le deuxième thème, celui de la (2) folie :

Dès le début de son séjour à Charenton, Sade fait face au phénomène de la folie :

"Quant à moi je vous assure que je n'en désire qu'un, c'est d'être (et cela le plus tôt possible) aussi loin de Charenton que j'en suis près, où néanmoins je serais fort aise d'avoir appris de vous, Monsieur, une très grande vérité contenue dans la lettre que vous venez de m'écrire, c'est qu'il y a beaucoup de gens qui ne se croient pas fous et qui le sont... Oh Monsieur quelle vérité ! » (D.A.F. Sade à M. de Coulmier, Charenton, ce 16 juillet 1803, 27 messidor an XI, (J.L.D., 488-490) in Laborde 1997 : 241)

En 1806, Sade écrit " au citoyen Gaufridy », son avocat, les « Souvenirs et nouvelles de la maison des fous » (Laborde 1997 : 264-268).

$\mathrm{Au}$ début de son enfermement à Charenton, Sade n'avait que le docteur Gastaldy à ses côtés, mais avec le temps, les relations de Sade avec le directeur, M. de Coulmier, s'améliorent de manière significative ${ }^{22}$.

Laborde identifie la « Dénonciation anonyme à l'encontre du citoyen Sade » comme étant celle de Royer-Collard lui-même contre Sade, mais aussi contre

22 Leurs relations améliorées, Sade demande à M. de Coulmier « de me laisser l'homme qu'il m'a donné et de ne pas me faire éprouver le désagrément, affreux pour moi, de changer tous les jours de visage " (octobre 1810, (B.N., MS. 24390, fo. 283 in Laborde 1997 : 333). Il exprime plusieurs fois ses désirs à M. de Coulmier (décembre 1810, (B.N., MS. 24390, fo. 281 in Laborde 1997 : 340) : « Je demande à M. de Coulmier :

1. D'être le maître de la clef de ma chambre, sauf à être fermée seulement à la première porte du corridor, de 10 heures du soir à 7 heures du matin ;

2. De me promener, sans être suivi aux heures qui me conviennent le mieux dans la journée, tant que le jardin est ouvert ;

3. De pouvoir causer librement avec les trois personnes désignées ci-dessous et ce, exclusivement, c'est-à-dire que je m'engage à ne parler à aucune autre personne : Mme Blotfière, ma voisine, M. de Savines, mon parent et M. de Léon ;

4. Enfin, que l'on me rende tout ce qui m'a été pris dernièrement tant en papier qu'en plume SADE»

Il écrit encore une lettre au sujet de ses désirs à M. de Coulmier (« 3 mars 1813, (B.N., MS. 24390, fo. 280) » in Laborde 1997 : 370) en demandant de « donner à Mme Quesnet la chambre d'au-dessus de moi » qu'il a fait espérer à son fils Armand. 
les méthodes de traitement favorisées par M. de Coulmier (« Paris, 20 août 1808 (A.N., F76294, \# 18, \# 19 » in Laborde 1997 : 278-279). Coulmier est le partisan de cette nouvelle idée de, non pas juste enfermer les malades, mais les guérir, et non pas par l'isolement et la séparation des sexes, comme Royer-Collard, mais par le théâtre, l'inclusion. Royer-Collard, par contre,n'y voit que de l'amusement, et pas un traitement.

«[...] Le docteur voit avec peine que M. de Sade jouisse dans cette maison d'une entière liberté ; qu'il communique avec les malades des deux sexes ; qu'il partage leurs promenades dans l'enclos qui leur est destiné. Il regarde comme un scandale public que cet homme immoral dirige un petit théâtre que le directeur de l'Hospice, M. de Coulmier a cru devoir autoriser dans l'intérieur pour l'amusement et la distraction des malades.

Il demande qu'on assigne à $M$. de Sade un autre lieu de réclusion afin que les malades qu'il traite ne reçoive pas sans cesse l'impression de la profonde corruption du détenu.

Observation :

M. de Sade détenu à Pélagie en a été retiré parce qu'il corrompait des jeunes gens qui se trouvaient alors dans cette maison de détention.

Transféré à Bicêtre, il a fallu l'en retirer pour le même motif et M. le Préfet, d'après les ordres de Votre Excellence l'a fait déposer à Charenton.

Le médecin qui le voit là avec peine, ne lui impute aucun des faits qui lui ont valu d'être éloigné de Sainte-Pélagie et de Bicêtre. D'après lui seulement, le bruit général, dans la maison de Charenton est qu'il vit avec une femme qui passe pour être sa fille (Mme Quesnet). Voilà une imputation vague.

Le souvenir de son ancien libertinage et l'espèce de supériorité qu'il exerce pour la direction du théâtre soit quant aux répétitions soit quant aux compositions : le scandale public qui en résulte, voilà les griefs. [...]

Il est possible que, dans sa demande, le docteur ait en vue de contrarier M. de Coulmier qui fait tourner à l'amusement des malades la vive imagination et l'esprit de M. de Sade. »

Dubois écrit un rapport le 2 septembre 1808 «(A.N., F7 6294, \# 16) » (Laborde 1997 : 280-282) au sénateur ministre de la Police générale dans lequel il rapporte avoir appelé le directeur de Coulmier à la Préfecture qui « dit même à cet égard qu'il a beaucoup d'obligation à de Sade, parce que regardant la comédie comme un moyen curatif de l'aliénation d'esprit, il se trouve heureux d'avoir dans son hospice un homme capable de former à la scène les aliénés qu'il veut guérir par ce genre de remède. » (Ibid. : 281)

Dubois y qualifie l'état de Sade comme « un état habituel de démence libertine » et propose son transfert «soit un Château de Ham soit dans toute autre prison d'État » (Ibid.) aux frais de la famille, ce qui a été approuvé, et même après l'intervention d'une partie de sa famille, la décision est maintenue (Ibid. : 283). Après une nouvelle intervention de la famille, et du docteur Deguise, ancien 
élève du docteur Gastaldy, favorable aux activités théâtrales de Sade, qui indique l'état de pléthore de Sade (« une goutte errante qui se porte à la poitrine », qui demande " des exercices et des promenades très modérées " ("Certificat du docteur Deguise en faveur du citoyen Sade, Charenton, 26 avril 1809, (A.N., F ${ }^{7}$ 6294, \# 12) », in Laborde 1997 : 285), dans la première moitié du mois d'avril est approuvé un ajournement du départ (1997 : 284).

\section{Le théâtre}

Au sujet du théâtre comme un moyen thérapeutique, construit à Charenton pour permettre aux malades de jouer la comédie, l'histoire commence avec le docteur Joseph Gastaldi, le médecin-chef de l'hospice de Charenton, que Sade connaît depuis l'époque où il vivait à Saint-Ouen, quand il l'a soigné d'une fistule. C'est lui qui va lui permettre de faire du théâtre, et de joindre ses intérêts en médecine avec ses aspirations humanitaires (que Sade a prouvé lors de la Révolution) (Laborde 1997 : 230) et ses rêves, qu'il avait essayé de réaliser déjà à La Coste et à Mazan en 1772, interrompus par l'affaire de Marseille. Avec la permission de Gastaldi et de Coulmier, Sade y devient metteur en scène, directeur du théâtre, acteur, écrivain et/ou adaptateur et décorateur pour la scène construite pour lui et sur ses plans (Ibid.). Son ton change, il n'y a plus de désespoir ou de fureur, et, après la mort du docteur Gastaldi (1805), le directeur Coulmier le soutient, car il comprend les avantages des représentations théâtrales pour la renommée de son hospice.

Les relations entre Coulmier et Sade, au début tendues (en témoigne la «Plainte de Sade contre M. de Coulmier, Charenton, 1804, (J.L.D., 493-494) in Laborde 1997 : 246 pour sa « conduite meurtrière envers » lui (ibid.)), vont devenir même cordiales grâce au théâtre.

La lettre que Sade écrit «A Mme Cochelet, Dame de la Reine de Hollande ${ }^{23}$, Spectacle du 23 mai 1810, (Revue Anecdotique, nlle. Série, $1^{\text {er }}$, 1860, 103-106) » (Laborde 1997 : 302), pour l'informer que, le 28 mai, ils donneront L'Esprit de contradiction, Marton et Frontin et Les Deux Savoyards, témoigne de l'intérêt qu'a suscité le théâtre de Charenton.

La lettre de Sade à M. de Coulmier, «Spectacle du 28 mai 1810 », où il lui demande d'approuver le répertoire, est accompagnée d'une « liste rectifiée par M. le directeur » qui spécifie les chiffres : les 90 places pour les invités (par noms), les 36 places pour les employés de la maison, les 60 malades, et le total de 186 places (Laborde 1997: 303-304). Ainsi Sade réussit non seulement d'introduire le théâtre comme moyen thérapeutique, mais d'yfaire joindre sous son égide le tout-Paris, le personnel et les malades.

A la fin de 1813, encore, il va essayer de réussir comme auteur dramatique en envoyant des manuscrits «Aux administrateurs du Théâtre-Français, fin 1813, (Vie

23 Reine de Hollande, Hortense de Beauharnais (1783-1837), fille du vicomte de Beauharnais et de Joséphine de Beauharnais, première épouse de Napoléon Bonaparte. Elle épousa Louis Bonaparte, le frère de Napoléon. Elle eut un fils, le futur Napoléon III. 
du., II, 216-217) » (Laborde 1997 : 372). Dans cette lettre il leur offre une tragédie en cinq actes, « lue à ce théâtre le 14 novembre 1791 et reçue à corrections » qui ont été faites. Il l'envoie une seconde fois en cet état ${ }^{24}$.

Quand, en septembre 1814, M. Roulhac du Maupas remplace M. de Coulmier, il écrit immédiatement une longue lettre, «M. Roulhac du Maupas, directeur de l'Hospice de Charenton à M. de Montesquiou ${ }^{25}$, ministre de l'Intérieur, Charenton, 7 septembre 1814, (B.N., MS. 24390, ff. 12-16) » in Laborde 1997 : 374383). Il y récapitule les péchés de ce prisonnier d'État : " maintenu en détention par décision signée Napoléon au bas des États présentés au Conseil privé des 19 avril et 3 mai 1812 » $1997: 377)$, il dénombre ses manuscrits, ceux qu'il a fait copier par d'autres malades, La Tour Mystérieuse, opéra comique en un acte, dont la copie n'était pas encore finie, et Le Magistrat Prévicateur, copiée depuis plusieurs mois ${ }^{26}$. Il s'aventure même à donner au ministre une critique de La Tour Mystérieuse qu'il a lue :

« un mauvais opéra sans sel, sans esprit, m'annonçant qu'un talent au-dessous du médiocre et dont la représentation ne provoquerait certainement que les baîllements et les sifflets ; ne portant point d'ailleurs le cachet de perversité et de dépravation dont on pouvait le supposer empreint comme d'autres ouvrages de l'auteur» $(1997: 378)$.

\section{Du roman libertin à la métaphorisation moderne : Métaphore 1}

Le caractère dit «théâtral » du récit libertin, patent depuis longtemps, et rendue flou à travers la métaphorisation, l'analogie, incitée à la fois par l'obsession théâtrale de l'époque, par les gravures des romans, par la performativité du texte composé de "scènes » à faire, à exécuter, est reconnaissable aujourd'hui notamment comme une préfiguration de la pornographie moderne à travers le caractère mécanique, spectaculaire (Polanz 2017 :278), et scopique de l'acte sexuel qui a lieu pour être une performance, un " montrer-faire », " showing down $^{27} \gg$.

24 Il avait fait quelques lectures particulières avant de le donner à lire au comité. Il leur dit d'avoir, en outre des petites pièces, encore « trois pièces de caractères, en cinq actes et en vers, dont l'une, reçue d'une voix unanime, m'a procuré mes entrées trois ans. (au Théâtre Français) ».

25 Abbé de Montesquiou, Fezensac, abbé François-Xavier, Marc-Antoine, duc de.

26 Sade écrit à M. Roulhac du Maupas (« 5 novembre 1814, (Arch. A. Bégis) 》 (Laborde 1997 : 388-389) en demandant la permission pour que M. Donge, chef du bureau de la Loterie, vient lire le journal à Mme Quesnet et à lui, et qu'il lui soit permis de continuer de copier les « ouvrages dramatiques reçus à différents théâtres et tous approuvés de la police ».

27 Richard Schechner, "From Ritual to Theater and Back", dans Performance Theory, London and New York, Routledge, 1988, p. 108. Version française : Performance : expérimentation et théorie du théâtre aux USA, dir. Anne Cuisset, Marie Pecorari et Christian Biet, trad. Marie Pecorari et Marc Boucher, Montreuil-sous-Bois, Éditions Théâtrales, 2008, in Polanz $2017: 278$. 
Le caractère nettement artificiel, mais qui pourtant mime la nature (reflet), féerique-merveilleux, baroque (décor «à l'italienne ») de cette théâtralité, notion problématique par soi-même, rendue célèbre par le dramaturge et théoricien Nikolai Nikolaevich Evreinov (« teatralnost ») ${ }^{28}$, laisse deviner que la théâtralité est un processus et non une essence, ni de la performance, ni du texte (Polanz 2017 : 280).

Or, à la théâtralité de Sade, mise en évidence par Annie Le Brun ${ }^{29}$, à la performativité du roman libertin, qu'on est censé lire d'une main, correspond aujourd'hui la métaphorisation de Sade, peut-être parce qu'il fait joindre le personnel, l'esthétique et le politique ${ }^{30}$, mais qui, dans ces deux avatars, détiennent aussi un caractère décidément grotesque et burlesque.

La première métaphorisation est la suivante : la métaphore de la société croate contemporaine en transition chez Ivo Brešan, écrivain, dramaturge, romancier, essayiste et scénariste croate (1936-2017), qui introduit la figure de Sade dans son roman Les Maudits (Prokletnici, Profil international, 2010), où il joue avec la réduction de Sade au sadisme, en opposant les penchants de Sade au sadomasochisme social d'un pays en transition, ce qui pourrait être dit le contraire même de sa situation en France. En France, le scandale de Sade gît dans sa "normalisation », dont témoignent le Sade up de Secka, et l'ouvrage de Noëlle Châtelet, et que remettent en question les œuvres récentes qui ont fait date comme celle d'Éric Marty et de Michel Delon.

Miran Šegota, metteur en scène âgé, trouve dans sa maison familiale à Senj l'autobiographie de marquis de Sade et apprend que ce dernier est son ancêtre. En remettant en question la sexualité dans le domaine personnel et public, Šegota essaie de mettre en scène (dans le théâtre «Sodome » qu'il construit, qui est finalement englouti par le feu) les pièces de Sade, pour « abattre les fausses valeurs de la société contemporaine », comme il dit, ce qui le mène à la folie. La

28 Nikolai Nikolaevich Evreinov, Teatr kak takovoi (Le Théâtre dans la vie) (St. Petersburg, Sovremennoe iskusstvo, 1912 ; Berlin, Academia, 1923, The Theater in Life, trad. Alexander I. Nazaroff, New York, Brentano's, 1927 in Polanz, Ibid. Il est connu surtout pour son essai Apologie pour la Théâtralité (1908) et sa devise : "To make a theatre of life is the duty of every artist. ... the stage must not borrow so much from life as life borrows from the stage."

29 La théâtralité, la scène « comme point de rencontre entre le réel et l'imaginaire, l'unique et le nombre, le spectaculaire et le secret » (Annie Le Brun, «Un théâtre dressé sur notre abîme », Le Magazine littéraire, n² 284, janvier 1991, p. 35-36) s'incarne chez Brešan dans la mise en abyme de la scène chez Miran : comme si le théâtre lui-même s'avère être insuffisant, et exige un passage à l'acte, à la fois le retardant et l'intensifiant, " mais en faisant voir par avance la théâtralité illusoire au-delà de laquelle il y a toujours une 'autre scène' » (Ibid.).

30 Il s'agit d'un clin d'œil au Marat/Sade de Weiss, car, comme l'indique Maurice Lever, il est inexact et abusif d'appliquer cette définition du «psychodrame moderne » aux essais dramatiques du marquis à Charenton - les malades ne jouaient pas un rôle adapté à leur psychose. Le drame de Weiss, créé le 29 avril 1964, au Schillertheater de Berlin, dans une mise en scène de Konrad Swinarski, est fondé sur ce postulat. 
vie de Šegota va commencer à ressembler à la vie de son ancêtre, et, à un certain moment, il va commencer à croire être Sade. La vie de Šegota, donc, devient théâtre, la mise en scène de la vie de marquis de Sade. Sa vie semble continuer la malédiction de ses ancêtres qui ont tous péri au moment où ils ont ouvert cette autobiographie. Brešan l'écrit, cette autobiographie non existante, mais Šegota, son personnage, va même la continuer ${ }^{31}$.

Et cependant, l'intention de Brešan gît ailleurs ; sa palinodie n'est pas foucaldienne - l'abjuration ne «prend » pas car le Sade de Brešan doit être, non pas «normalisé », mais "typé ». Sa jeunesse débauchée se transforme en une suite d'anecdotes drôles et invraisemblables, qu'il vit presque " par accident ». Le Sade de Brešan est un maladroit. Même si ce Sade-là déçoit par son caractère « antimoral » qui fait toujours partie de la morale ${ }^{32}$, Brešan ne vise pas la disculpation - la société en fait un Candide. Brešan emploie sa vieille recette, comme dans sa pièce culte, La Représentation de 'Hamlet' au village de Mrduša-d'enbas-1'immixtion de la fiction dans la réalité devenue fiction historique hypocrite, pour souligner le contraste entre la France du XVIII ${ }^{\mathrm{e}}$ siècle et la Croatie du XXI ${ }^{\mathrm{e}}$ siècle, et subvertir l'image du monde contemporain ${ }^{33}$.

Ainsi Brešan promeut-il l'insoumission existentielle de Sade-Miran passe au statut de fou, grâce au diagnostic du psychiatre charlatan, M. Žigman, Hochstapler qui, dans ce monde kafkaïen, rappelle le Vespoli de L'Histoire de Juliette et invente une méthode $\mathrm{du}$ « choc résocialisateur ».

Le dernier coup de théâtre de Šegota : tout au long du roman, il « jouait » le fou. L'idée de Brešan, d'opposer les préférences de Sade au sadomasochisme social ex-yougoslave introduit cette différence essentielle entre la folie «jouée » et

31 Chez Brešan, Sade tombe vraiment et fatalement amoureux d'Anne-Prospère, sa bellesœur, et s'enfuit avec elle avant son mariage, tout comme chez Wright, de la jeune Madeleine. Cet amour romantique (décidément « moderne ») et le drame de l'arrestation à Lyon provoque sa « rage », et le transforme en un personnage du roman psychologique, ce que Foucault rejette et trouve inintéressant dans son Introduction à Rousseau juge Jean-Jacques. Dialogues. Sade, tout « balkanisé » qu'il est, devient « logique », son histoire avec Renée-Pélagie l'histoire d'un « vrai couple », modèle d'un intime trop contemporain (faute de passions, il y a l'attachement, ce qui est une compréhension quelque peu simpliste de l'intime), et même ses penchants, qui se réduisent plus au masochisme qu'au sadisme, se trouvent rationnalisés par l'impuissance, l'âge et l'équilibre épicurien entre la douleur, le plaisir/la jouissance et sa satisfaction.

32 Cf. Nietzsche, La Généalogie de la morale, 208, et Barthes, FDA, CEuvresComplètes, V, Paris, Seuil, 2002, p. 218-221 (figure «L'obscène de l'amour »).

33 Ainsi Brešan inclut-il dans son roman les faits divers authentiques de la presse croate des vingt dernières années que Šegota collectionne, des crimes de guerre, des massacres et des viols, jusqu'aux crimes passionnels de l'époque de la guerre ou de l'après-guerre. Et tout ce que Sade a dit et vécu ne semble qu'un mauvais spectacle, même la guillotine, que le libertin compare à une pièce de théâtre pleine des tirades superflues des bourreaux et des victimes. En revanche, son engagement politique est pris au sérieux: il est un anarchiste convaincu qui professe la loi de la nature (et non pas le mal en tant que tel), et rejette la dévotion, la fétichisation et la religiosité sous toutes ses formes. Il ne s'apaise, dans la vision de Brešan, que vers la fin de ses jours à Charenton. 
la « vraie » folie qui correspond à la thèse fondamentale du roman sur l'horreur de l'époque moderne, inouie et lisible dans Auschwitz, Jasenovac et Srebrenica. À la différence de Sade, Miran n’apparente pas le théâtre à une « réhabilitation » sociale et à la gloire ${ }^{34}$. Par ailleurs, cette différence entre la folie « figurée » et la « vraie » folie de l'époque moderne (la guerre) est attendue, car la folie de Miran reste « extérieure », fonctionnelle, elle n'a rien de la « déraison sadienne » foucaldienne. Brešan se tient du côté de la figure blanchotienne de la pure contestation. Son Sade est à la fois « engagé », décidément politique et « moral », et émotionnel, même sentimental dans le sens moderne du terme. Il n'y a que le rire et la catharsis finale qui « sauvent le jour ». Des innombrables coups de boutoir que Brešan et Šegota distribuent à la ronde avec un esprit endiablé témoignent de leur quête de la provocation, du blasphème - Brešan, lors de la conversation téléphonique avec nous, répète ce qu'affirme Šegota à la fin du roman, en citant Oscar Wilde, et son essai « The Soul of Man under Socialism ${ }^{35}$ »- peu importe si quelque chose est arrivé ou non : il choisit de porter le dernier coup au concept du réel et de l'imaginaire ${ }^{36}$. Les Maudits se terminent par le succès de la pièce de Miran avec des fous qui improvisent à l'asile de l'île d'Ugljan.

34 CEuvres complètes du Marquis de Sade, Paris, Au Cercle du Livre Précieux, 1967, t. 11, Correspondance, lettre CLXXIX, p. 441-442.

35 « In its development it will be assisted by Christianity, if men desire that; but if men do not desire that, it will develop none the less surely. For it will not worry itself about the past, nor care whether things happened or did not happen. Nor will it admit any laws but its own laws; nor any authority but its own authority. Yet it will love those who sought to intensify it, and speak often of them. And of these Christ was one. » (Oscar Wilde, The Soul of Man under Socialism, 1891)

36 D' « Emmelet ", revu et corrigé, à Mrduša, village fictif de l'arrière-pays dalmate, et sa tragi-comédie burlesque, sa farce grinçante et sa satire politique pessimiste - une double mise en abyme, de l'œuvre de Shakespeare, et de la société yougoslave, avec les dirigeants du comité local du Parti communiste et les villageois devenus acteurs, qui nourrissent ce comique terrible et grotesque à la scatologie baroque (rappelant Le Prévaricateur, ou Un magistrat du temps passé (1783) de Sade), au Sade "balkanisé », réintégré par l'imaginaire à la société croate de l'après-guerre, l'œuvre de Brešan exploite la mise en abyme et souligne la force pratique de la représentation théâtrale qui n'est pas, chez lui, tant une illusion (comme dans L'Union des arts de Sade) qu'une dénonciation, une subversion de l'ancienne Yougoslavie, une métaphore de la bigoterie générale, une ironisation des « sections culturelles » de l'époque (un clin d'œil à la Section des Piques de Sade), qui fait que la bêtise devient leur fort. À un profiteur, La Chope, le président du comité local, qui détourne les fonds de la coopérative, sous le masque du faux tyran fratricide (roi fourbe Claudius) correspond l'autre, le psychiatre charlatan Žigman ; aux paysans abrutis, égoïstes, misogynes, copulateurs, gourmands, gueulards, qui révèlent la fourberie de cette caste des apparatchiks de campagne, définitivement irrécupérables, correspond Šegota, l'intellectuel. Si la critique du système socialiste chez Brešan semble quelque peu simple aujourd'hui, il faut se rappeler que la pièce date d'une autre époque. Vu son caractère profondément dénonciateur, la compréhension de cette œuvre dépend foncièrement d'une bonne traduction. Et même plus quand il s'agit d'une pièce de théâtre que d'un roman. Sinon, la satyre tombe à plat, l'humour se perd, la critique n'est plus visible, et la pièce ressemble à une fable. 
En rejoignant la définition foucaldienne de la fiction historique, Brešan achève le roman par une citation de L'Erotisme de Bataille ${ }^{37}$, qui devrait témoigner de sa capacité de voir Sade « objectivement». Et cette citation célèbre refuse de réduire la pensée de Sade à la folie. Elle la nomme « excès vertigineux », « excessif sommet de ce que nous sommes », en rejetant la vie des « ombres apeurées » et rappelant la devise de Juliette : «Le passé m'encourage, le présent m'électrise, je crains peu l'avenir». Bref, Miran a toujours su qui est-il, et qui est Sade ; les hallucinations n'étaient que l'effet des médicaments.

La dernière apparition de Sade en Croatie fait voir que Sade n'y est pas ni dédiabolisé - le renouveau catholique ambiant ne l'a pas permis -, ni « normalisé ». Le romanesque y permet de faire joindre le théâtre de Sade, la question de sa mise en scène, de la folie qui y règne, et des « restes » des interprétations, tout en y réintroduisant les enjeux contemporains, notamment sociaux (les souvenirs de la guerre récente en Croatie et en Bosnie, entre autres). Le théâtre serait donc tout d'abord une scène, l'un des lieux qui fait naître quelque chose de plus « sadien » que Sade lui-même.

La vie devient scène, la scène est vie et devient scène de la vie. Le « traitement moral » de la folie de M. de Coulmier (docteur Tomislav Steinbach chez Brešan) et du docteur Gastaldy, effaçant les frontières entre la raison et la folie (ce qui les rend inadmissibles au professeur Esquirol ${ }^{38}$ ), est confirmé par la vie de Miran, et réintroduit Momus, le dieu de la folie (chez Sade dans L'Hommage de la reconnaissance), léger et optimiste, comme un faire-valoir de l'institution aliéniste, et non pas comme un agent de la satire (Dangeville 1999 : 495). Sade et Brešan

Dans le cas de Mrduša, qui a été traduite en français en 2009 (L'Espace d'un instant), par Johnny Kundid, avec la collaboration de Paul-Louis Thomas, il fallait peut-être rendre ce langage par ce qu'il représentait et représente en croate - un mélange étrange du dialecte (peut-être avec les Marseillais - la vogue des Ch'tis l'a rendue possible) et de la langue de bois d'un Maurice Thorez dans sa veine staliniste, ou de l'Humanité des années 50 avec leur «Budapest retrouve le sourire » le 4 novembre 1956, le jour où l'Armée Rouge entre en Hongrie. Rappelons-nous que la pièce elle-même a été écrite en 1965, et a été mise en scène pour la première fois en 1971, l'année du " printemps croate ». Sinon, même avec toute cette parodisation de Shakespeare, que les traducteurs rendent bien, l'effet est bien faible. Et toutes les lignes décasyllabiques, les interjections et la phraséologie « modernisée » n'y peuvent rien. Dans le cas des Maudits, l'effet serait différent, mais la tâche du traducteur encore plus dangereuse, étant donné qu'il s'agit d'une réappropriation d'un auteur français dans un autre contexte, différent, plus politique que culturel. Il serait curieux de suivre l'accueil d'un tel roman.

37 « De toute façon, la pensée de Sade n'est pas réductible à la folie. C'est seulement un excès, c'est un excès vertigineux, mais c'est l'excessif sommet de ce que nous sommes. De ce sommet, nous ne pouvons nous détourner sans nous détourner de nous-mêmes. Faute de nous rapprocher de ce sommet, de nous efforcer d'en gravir au moins les pentes, nous vivons comme des ombres apeurées - et c'est devant nous-mêmes que nous tremblons. » (Georges Bataille, L'Érotisme)

38 Marquis de Sade, CEuvres complètes, t. 15, Théâtre, t. 3, Rapport statistique du Pr. Esquirol, 1818, p. 494-495. Hippolyte de Colins partageait son opinion (thèse de Jean-François Reverzy, «Les journées de Charenton (ou l'asile dévoilé) »). 
confirment l'origine passionnelle de la folie (dans L'Hommage) et le bien-fondé $\mathrm{du}$ « traitement moral ${ }^{39}$ », à l'époque, une thérapie décidément moderne ${ }^{40}$. Et avec Brešan, Sade, " croatisé » et « barbarisé » de l'intérieur, même « typé », détient son scandale durable en trahissant par le moderne.

\section{Métaphore II}

La deuxième métaphorisation sous-entend une métaphore de l'écriture, celle de Quills de Doug Wright, mis en scène pour la première fois à New York en 1995, et publiée en $2005^{41}$. Wright choisit la confiscation des plumes de Sade (Ibid. : 171 et sq.) à Charenton, en 1807, pour une pièce de théâtre avec, selon l'auteur, une histoire simple : un jeu, une compétition entre les censeurs et Sade qui mène à l'absurde (lbid. : XX). S'y joint le thème de la folie et de son traitement, transformant la pièce en une «scène » de la folie dans le sens ranciérien du terme ${ }^{42}$, dont le

39 Sade arguait pour la défense du théâtre dans une lettre du 4 mai 1811 à sa parente, Mme de Bimard - qu'il permettrait de guérir de nombreux malades (Marquis de Sade, CEuvres complètes, t. 15, Théâtre, t. 3, p. 517).

40 M. le Coulmier, Précis sur la maison de santé de Charenton, dans Maurice Lever, Sade, p. 607.

41 Dans l'introduction, «Willful misbehavior » (2005: IX-XXII), Wright motive son choix d'écrire sur les provocateurs, « outsiders » excentriques qui subvertissent l'ordre d'une manière «morale », ce qui veut dire pour lui éthique (2005 : XI). Selon lui, les marginaux disent les vérités les plus humaines, notamment les artistes (Ibid. :XII). Et Sade, comme il dit, « in the pantheon of miscreants, the Marquis de Sade is the grandaddy of 'em all » (Ibid. : XVIII), bien qu'il motive aussi son choix de prendre le rapport de l'abbé de Coulmier, médecin, et Sade, l'écrivain fou, en y fourrant des éléments d'un roman à clef personnel (Ibid.).

${ }^{42}$ L'Empire devenu l'empire des passions introduit ici une certaine lecture, celle de Rancière, de deux concepts et le glissement de l'un à l'autre : le concept du dispositif, qui est pour Rancière une fabrication, et le concept de la scène, qui est pour lui une rencontre (2018: 30). Le « dispositif » foucaldien, dans l'acceptation de Rancière, produit un sujet assujetti, « quelque chose comme l'appareil qui impose la manière dont on va se placer, dont on va être identifié et devoir regarder et être vu » (Ibid.), « du côté de la conception heideggérienne de ce qui machine les places, la vision, les possibilités d'énoncer » (Ibid.), qui a un « noyau qui renvoie à la machination » (Ibid.) : « voilà ce qui produit ce que vous percevez et ce que vous pensez » (Ibid. : 31). Au contraire, la scène est le « lieu d'une rencontre qui est toujours aléatoire et soumise à un remaniement », « le moment où les choses peuvent basculer ». «Il y a subjectivation pour moi quand il y a reconfiguration des coordonnées d'un champ d'expérience. Et c'est ce qui est en jeu dans la scène » (Ibid.). Rancière (dans Les Scènes du peuple. Les Révoltes logiques, 1975-1985, Lyon, Horlieu éditions, 2003), en thématisant la censure et la surveillance des théâtres, choisit de s'éloigner des thèmes de la disciplinarisation pour montrer "que des bourgeois, des ministres, des policiers ne sachant pas très bien où était le problème, étaient confrontés à un type d'espace où le partage n'est pas évident et où en même temps on ne peut jamais prévoir les effets » (2018:41). La correspondance de Sade semble le confirmer (voir Dubois). Or, Rancière introduit une lecture spécifique de la subversion, différente de celle de Wright : en insistant « non seulement sur 
caractère remet en question le « pouvoir psychiatrique » foucaldien : Royer-Collard affirme (Ibid. : 237) que ce ne sont pas eux, mais lui, qui gouvernent à Charenton ${ }^{43}$.

Ainsi Wright semble-t-il suivre les définitions foucaldiennes de l'hôpital comme «un lieu ambigu : de constatation pour une vérité cachée et d'épreuve pour une vérité à produire », « une action directe sur la maladie », de révélation (pour le médecin) et de l'éclosion, de la production de la maladie (Foucault 2003 : 341). Charenton rentre dans la définition foucaldienne de l'hôpital du XVIII ${ }^{e}$ siècle, qui « était censé créer les conditions pour qu'éclate la vérité du mal », comme « un lieu d'observation et de démonstration, mais aussi de purification et d'épreuve » (lbid. : 342).

Le conflit entre les deux, M. de Coulmier et Dr. Royer-Collard, raconte l'antagonisme et ce que Rancière appelle la barrière : la folie avant le XVIII siècle, « pas systématiquement internée », « considérée comme une sorte d'erreur ou de l'illusion » que l'on soignait par deux lieux thérapeutiques : la nature, « la forme visible de la vérité » (Ibid. : 343), « le voyage, le repos, la promenade, la retraite, la coupure avec le monde artificiel et vain de la ville » et le théâtre, « nature inversée » (Ibid. : 344).

Chez Sade, et chez Brešan et Wright, survient une modification cruciale : pour paraphraser Foucault (Ibid.), on ne lui joue pas la comédie de sa propre folie, on ne la met pas en scène (d'ailleurs, la censure oblige), on ne lui prête pas une réalité fictive par les décors et les déguisements, pour lui faire apparaître la vérité ; c'est lui qui va construire un théâtre, écrire et mettre en scène des pièces que tout Paris va voir.

Foucault identifie ce moment de la modification au début du XIX ${ }^{\mathrm{e}}$ siècle, quand la folie ne relève plus de l'erreur, mais de l'aberration, de l'égarement, de l'écart par rapport à la conduite « régulière et normale »; « non plus comme jugement perturbé, mais comme trouble dans la manière d'agir, de vouloir, d'éprouver des passions, de prendre des décisions et d'être libre ; bref, [...] non plus sur l'axe véritéerreur-connaissance, mais sur l'axe passion-volonté-liberté ; moment de Hoffbauer et d'Esquirol ». Apparaît donc ce désir de «normaliser » les « anormaux ».

Sade pourrait être dit l'un de ces patients, dont la "volonté malade » fait face à la "volonté droite du médecin », Royer-Collard, qui exige la soumission : «Un processus, donc, d'opposition, de lutte et de domination » (Ibid. : 345) ${ }^{44}$.

l'espèce d'indécision dans la circulation de la parole, mais sur le fait que la subversion des identités et des places n'est pas liée à une espèce de concentration d'un camp sur sa logique propre, mais au contraire à l'indécision d'une limite », «le vacillement d'une 'barrière' » (Ibid. : 43), la barrière étant « un lieu de rencontre en même temps que de séparation » (Ibid.). Rancière conçoit la subversion comme la "rencontre entre des paroles, des rhétoriques, des cultures, des modes d'être », «la constitution d'espèces de mixtes », « dans cette zone frontière indécise » (Ibid. : 43).

43 Les techniques que mentionnent Foucault, l'isolement, l'interrogatoire, les traitementspunitions, les entretiens moraux (encouragements ou remontrances), les récompenses, les rapports préférentiels ont pour fonction de transformer le médecin en un " maître de la folie» (Ibid. : 345), qui jouit de son « surpouvoir» (Foucault 2003 : 346).

44 Ainsi «plus encore qu'un lieu de dévoilement, l'hôpital dont Esquirol a donné le modèle est un lieu d'affrontement ; la folie, volonté troublée, passion pervertie, doit y rencontrer une volonté droite et des passions orthodoxes » (Ibid. : 345). 
Déjà les premières lignes de la première scène introduisent la notion de la métaphore réussie (l'architexte M. Prioux la proclame «stirring and apt») qu'emploie Dr. Royer-Collard : «Charenton is my France, and I am her Napoleon » (2005 : 165).

Royer-Collard dénigre M. de Coulmier qu'il dit avoir ôté tous les instruments disciplinaires (« wicker dummy », mannequin en osier, « wire cage, and the straitjacket » Ibid. : 172), pour introduire « les interludes musicaux, exercices de l'aquarelle, et même Marivaux » (Ibid.). La conversation entre Coulmier, dr. Royer-Collard et le Marquis de Sade (scène 3) introduit le conflit entre deux concepts de la médecine/psychiatrie. Coulmier pose la question comment un traitement inhumain peut-il produire un comportement civilisé (Ibid. : 175). Il le veut « walking placard ( « human billboard » ou « homme-sandwich ») pour les stratégies humanitaires dans les hôpitaux, les asiles et les prisons (Ibid. : 176).

Tandis que dr. Royer-Collard le considère comme un « enfant errant », et non pas un homme (Ibid. : 181), Coulmier refuse de perpétuer les horreurs mêmes que le Marquis décrit si laborieusement (Ibid.).

Sade problématise l'impératif moral pour la littérature, que Coulmier veut assurer à travers la catharsis (Ibid. : 191). Coulmier définit sa maladie comme « libertine dementia » (Ibid.).

En annonçant qu'il doit confisquer son papier et sa plume, Sade dit qu'ils sont tout ce qu'il a, que c'est son seul salut, d'évacuer/décharger ses fantaisies sur le papier :

" Mon writing is involuntary, like the beating of my heart ! My constant erection ! I can't help it ! » (Ibid. : 193). Selon lui, ses romans ne sont pas les symptômes de sa folie, mais ils lui permettent de garder sa santé mentale (Ibid.).

La scène 7 annonce la marche inévitable vers l'absurde : les draps, les rideaux et les serviettes bordeaux signifient que le Marquis a commencé à écrire avec du vin sur ses draps. Il commence à écrire avec son sang sur sa chemise (scène 9) et transforme ses vêtements en texte (Ibid. : 205). Coulmier lui ôte ses vêtements et sa perruque, mais le Marquis affirme que sa plume la plus vigoureuse se trouve entre ses cuisses (scène 10, Ibid. : 209). La littérature devient résistance : il va raconter ses histoires à son voisin pour qu'il les transfère à son tour, « sa vision odieuse filtrée à travers les esprits des fous », qui pourraient les améliorer (scène 11, Ibid. : 212). Dr. Royer-Collard refuse de considérer « l'incident » comme une émeute, mais plutôt une escarmouche (Acte II, scène 1, Ibid. : 213), alors que Renée-Pélagie l'assure que l'histoire du Marquis est en train d'atteindre Paris, « comme une bête carnivore, bossue » (Ibid. : 219).

Accusé d'être coupable de la mort de la jeune Madeleine, que commet Bouchon, inspiré par l'histoire du Marquis, Coulmier lui annonce sa punition : le chirurgien va lui enlever la langue (Ibid. : 228) que Coulmier apporte au dr. RoyerCollard dans une petite boîte comme preuve (scène 5, Ibid. : 231). Royer-Collard lui annonce que la cellule du Marquis est devenue une «fantasmagorie des mots » (Ibid. :232). Le Marquis a craché dans ses excréments pour obtenir de l'encre. Il a « décoré sa chambre avec du langage » (Ibid.). Bien que ses lèvres sont cousues, on entend sa voix racontant une histoire où il n'y a pas de débauche (Ibid. : 233). 
Dr. Royer-Collard ne croit pas, il pense que Marquis les a trompés « en masquant son obscénité par la métaphore »; selon lui, le Marquis est devenu si habile qu'il ne laisse que des phrases vagues pour que le lecteur les remplissent avec des détails salaces. « The story ripples with innuendo ! ». Royer-Collard va loin avec l'interprétation : «Choose any line of text, and beneath its harmless veneer you'll find nothing but sin and degeneracy ! Go ahead ! I dare you ! (...) It's all there, Abbé ! Lurking beneath his seemingly flaccid prose !» (Ibid. : 235).

Royer-Collard veut donner sa démission, fermer Charenton et libérer les patients pour qu'ils propagent la philosophie du Marquis, mais Coulmier réussit à obtenir une dernière chance (Ibid. : 238).

Ici le paysage change ; ce n'est plus la métaphore, une certaine métaphore, qui est remise en question, mais LA métaphore, le concept même de la métaphore, qui fait voir la FOLIE de la métaphorisation, de l'inscription, de la surinterprétation, en transformant cette pièce en une «scène » :

« La vérité de l'histoire gît entre les lignes, et non dans les lignes » (Coulmier, scène 6, Ibid. : 239).

Coulmier apporte à Royer-Collard les preuves, les boîtes contenant ses extrémités et sa verge, qu'a enlevé le boucher (et non pas un chirurgien, en tant que punition), reconnaissant d'avoir trouvé une certaine satisfaction dans ces procédures (avec chaque coup de hache, il pensait qu'il s'approchait de Dieu, scène 7, Ibid. : 241). Selon Royer-Collard, Coulmier doit, non seulement casser son corps qui ressemble à une «pitoyable étoile de mer » (Ibid. : 242), mais briser son esprit. Renée-Pélagie informe Royer-Collard (scène 10, Ibid. : 247) que sa réputation est sauvée, non pas parce qu'ils ont réduit au silence la muse du Marquis, mais, au contraire, parce qu'ils l'ont transformé de monstre en martyr (Ibid. : 248). Marquis est dit être « démonté », et mis en 7 boîtes. Elle proteste : «I bade you kill the author - not the man ! (Ibid. : 249). La médecine signe sa capitulation ; Royer-Collard se distancie de Coulmier devenu « monstre » (beast) et de ses méthodes.

Coulmier se justifie par ses bonnes intentions ${ }^{45}$ et se repent par la mortification qu'il ne peut pas arrêter ${ }^{46}$. Royer-Collard veut publier les manuscrits du Marquis, anonymement, pour sauver Charenton, comme le pendant de la Bible (Ibid. : 256). Ils veulent faire cadeau de la tête du Marquis au laboratoire phrénologique, pour prouver qu'ils ne sont pas les mêmes, qu'ils ne sont pas monstres.

Dans l'avant-dernière scène $\left(12^{\mathrm{e}}\right)$, Coulmier, devenu fou, dans sa cellule, appelle Valcour et revendique du papier pour écrire. Dans la dernière, $13^{\mathrm{e}}$ scène, les parties du corps du Marquis se relèvent des sept boîtes, et trouvent du papier

45 «Slander! Perjury! Lies! Let them judge me by my intentions, not by the acts themselves. Violence in pursuit of pleasure is one thing. In pursuit of justice, it's another » (Ibid. : 253).

46 « My body has become a map of suffering, and I am its obsessed cartographer. I have stared into the face of evil, Doctor. And.... Heaven help me, please... I have never seen such terrible beauty. Who have I become ? What am I to do in my defense ? » (Ibid. : 254) 
et une plume. La tête du Marquis dicte l'histoire du vertueux M. de Coulmier qui a rencontré un coquin qui avait l'habitude d'écrire des histoires. Coulmier revendique une plume et la tête du Marquis rit. Le rire du Marquis et des fous clôt la pièce.

Le chamboulement du concept de la métaphore chez Wright assure que, face à l'image d'une vérité à dévoiler derrière les apparences (métaphore), les deux espaces, l'asile et le théâtre, constituent ici une scène ${ }^{47}$ polémique qui introduit une conceptualisation de l'empire des passions (Rancière 2018 : 14), non pas l'apparence, mais « la scène de la manifestation », non pas une arrière-scène, mais la construction d'un univers d'apparences où la théâtralité joue la présentation de ce qui apparaît. Cette scène est paradoxale car elle réussit à universaliser l'expérience du Sade de Wright, celui qui est le singulier même.

Cette scène est le produit d'une «mutation du regard » qui crée un « dissensus ${ }^{48}$ » (né de la «mésentente ») (Ibid. : 23) entre la mise en scène de l'asile de fous devenu théâtre dans plusieurs sens du terme et celle de la folie de la métaphorisation et de la surinterprétation. Wright montre que les « rituels maniaques » de ces gens « sont des actions » (Rancière 2018 : 63). «"Til Madness Do Us Part ${ }^{49} »$.

\section{Coda}

L'Empire devient l'empire des passions à Charenton et la scène de cet empire, biographique, historique, littéraire, métaphorique, en remettant en question la métaphore même, fait voir comment les concepts signifient tout en exposant l'effilement qui les habite. Sade devient ici événement ${ }^{50}$. Cet empire des passions, au sein de la folie et de la folie des métaphores qui ne se pétrifient pas, qui ne font que se multiplier dans leur inévitable accélération, déploie une possibilité, d'y

47 «Travailler sur la scène, c'est refuser toute une logique de l'évolution, du long terme, de l'explication par un ensemble de conditions historiques ou du renvoi à une réalité cachée derrière les apparences » (Rancière 2018 : 11) « La scène, pour moi, est le lieu où le concept se divise en s'exposant. » (Ibid. : 12)

48 «Le dissensus, fondamentalement, est l'écart entre deux mises en scène sensibles (...) des mises en scène différentes de la présence des sujets collectifs quant au sens même de cette présence » (Rancière 2018 : 25) Le dissensus n'est pas simplement un écart ou un scandale qui rompt le consensus (Rancière $2018: 68$ ) : « il s'agit de la capacité d'insérer l'écart dans la construction d'une autre forme de sens commun » (Ibid.).

49 Le titre éponyme du documentaire chinois de Wang Bing, traduit en français comme «A la folie » (titre original : Feng ai, traditionnel : 瘋愛, simplifié : 疯爱) sorti en 2013 (Mostra de Venise), et en France en 2015. Il s'agit d'une histoire des pensionnaires d'un asile d'aliénés/d'un hôpital psychiatrique du Yunnan (Sud-Ouest de la Chine), suivis pendant trois mois, notamment les 50 de l'étage pour homme et un patient en permission de sortie et de retour chez ses parents.

50 «L'événement n'est pas un donné. Ce qui fait événement, c'est la scène. » (Rancière 2018 : 123) «Il y a événement conceptuel là où on peut construire un microcosme. » (Rancière 2018: 124) 
insérer, si ce n'est que rarement, la littérature, mais celle qui se tiendrait toujours, par définition, en marge, en dehors même du dépassement des concepts binaires du centre et de la périphérie.

« Literature is, I believe, a very safe cage for the beast within » (Wright 2005 : XXII). Si la métaphore tue, la scène fait naître. Et le manque de complément de cette phrase dit tout.

\section{Bibliographie choisie :}

ANGREMY, Annie, "Les manuscrits de Sade à la Bibliothèque nationale », dans Béatrice Didier et Jacques Neefs (éd.), La Fin de l'Ancien Régime, Sade, Restif, Beaumarchais. Manuscrits de la Révolution I, Saint-Denis, Presses Universitaires de Vincennes, coll. « Manuscrits modernes », 1991.

BREŠAN, Ivo, Predstava Hamleta u selu Mrduša Donja, Zagreb, Sysprint, 2000.

BREŠAN, Ivo, Prokletnici, Zagreb, Profil international, 2010.

CRAVERI, Benedetta, Les derniers libertins, Paris, Flammarion, coll. « Au fil de l'histoire », 2016.

DANGEVILLE, Sylvie, Le Théâtre change et représente, Lecture critique des œuvres dramatiques de Sade, préface de Maurice Lever, Paris, Honoré Champion Editeur, 1999 ;

FOUCAULT, Michel, Les Anormaux, Cours au Collège de France. 1974 - 1975, Paris, Seuil/Gallimard, 1999 (Hautes Etudes).

FOUCAULT, Michel, Préface à la transgression, Hommage à Georges Bataille, suivi de Ceci n'est pas une préface de Francis Marmande, Paris, Gallimard 1994/ Nouvelles Éditions Lignes, 2012.

FOUCAULT, Michel, Le Pouvoir psychiatrique, Cours au Collège de France. 1973-1974, Paris, EHESS/Gallimard/Seuil, coll. Hautes Études, 2003.

GOULEMOT, Jean-Marie, Ces livres qu'on ne lit que d'une main. Lecture et lecteurs de livres pornographiques au XVIIIe siècle, Aix-en-Provence, Alinéa, 1991.

GUENIFFEY, Patrice, Le Dix-huit Brumaire, L'épilogue de la Révolution française (9-10 novembre 1799), Paris, Gallimard, collection Folio histoire, 2008.

LABORDE, Alice M., Correspondances du Marquis de Sade et de ses proches enrichies de documents notes et commentaires, tome XXV (1798-1814), Les dernières années, Genève, Éditions Slatkine, 1997.

LE BRUN, Annie, «Un théâtre dressé sur notre abîme », Le Magazine littéraire, n 284, janvier 1991, p. 35-36.

LEVER, Maurice (dir.), Bibliothèque Sade I. Papiers de famille. Le règne du père (17211760), Paris, Fayard, 1993.

LEVER, Maurice (dir.), Bibliothèque Sade II, Papiers de famille. Le marquis et les siens (1761-1815), Paris, Fayard, 1995.

LEVER, Maurice, Donatien Alphonse François, marquis de Sade, Paris, Fayard, 1991.

LEVER, Maurice, éd., " Je jure au marquis de Sade mon amant de n'être jamais qu'à lui... », Paris, Fayard, 2005. 
LEVER, Maurice, éd. «Quatre lettres inédites d’Ange Goudar au Marquis de Sade », Dix-Huitième Siècle, 1991, n²3, p. 223-232.

PEROVIC, Sanja, «Sade's Theatre : Pleasure, Vision, Masochism (review)», French Studies : A Quarterly Review 65, n²1, Oxford University Press, 2011, p. 97-98, accessed January 26, 2013 from Project MUSE database (http://muse.jhu.edu/).

POLANZ, Dorothée, "Sexe, performance et techniques de spectacle dans le récit libertin », in Dix-huitième siècle (revue annuelle), Société du spectacle, n 49, La

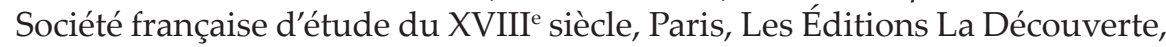
2017,p. 277-290.

QUIGNARD, Pascal, L'enfant d'Ingolstadt, Dernier royaume X, Paris, Bernard Grasset, 2018.

RANCIERE, Jacques, avec Adnen Jdey, La Méthode de la scène, Paris, Lignes, 2018.

SADE, Donatien Alphonse François, marquis de, Anne-Prospère de Launay : «L'amour de Sade », lettres retrouvées et éditées par Pierre Leroy, avant-propos de Philippe Sollers, Paris, Gallimard, 2003.

SADE, Donatien Alphonse François, marquis de, Les Cent-Vingt Journées de Sodome, Aline et Valcour, Paris, Gallimard, Bibliothèque de la Pléiade, 1990.

SADE, Donatien Alphonse François, marquis de, Correspondance inédite du Marquis de Sade, de ses proches et de ses familiers publiée avec une introduction des annales et des notes, Paris, Libraire de France, 1929 (éd. P. Bourdin)

SADE, Donatien Alphonse François, marquis de, Histoire de Juliette, dans : CEuvres complètes du Marquis de Sade, t. IX, Paris, Éditions Pauvert, 1987.

SADE, Donatien Alphonse François, marquis de, Idées sur les romans, in : Les Crimes de l'Amour, Paris, Union générale des Éditeurs, (10/18), 1971.

SADE, Donatien Alphonse François, marquis de, Les Infortunes de la Vertu, Paris, Club des Classiques, 1977.

SADE, Donatien Alphonse François, marquis de, Lettres à sa femme, Arles, Actes Sud, 1997 (éd. Marc Buffat).

SADE, Donatien Alphonse François, marquis de, Lettres et Mélanges littéraires écrits à Vincennes et à la Bastille, Paris, Borderie, 1980, tome II (éditeurs Georges Daumas et Gilbert Lely).

SADE, Donatien Alphonse François, marquis de, Lettres inédites et documents retrouvés par Jean Louis Debauve, Paris, Ramsay / Pauvert, 1990.

SADE, Donatien Alphonse François, marquis de, CEuvres, édition établie par Michel Delon, Paris, Gallimard, coll. «Bibliothèque de la Pléiade »,1990-1998, 3 tomes.

SADE, marquis de, CEuvres complètes, tome XIII-XV, Théâtre, tome I-III, Paris, Pauvert, 1991.

SADE, Donatien Alphonse François, marquis de, Voyage à Naples, Paris, Payot \& Rivages, 2008 (éd. Chantal Thomas).

SADE, Donatien Alphonse François, marquis de, Voyage d'Italie ou Dissertations critiques, historiques et philosophiques sur les villes de Florence, Rome, Naples, Lorette et les routes adjacentes de ces quatre villes, Paris, Fayard, 1995 (édition établie et présentée par Maurice Lever) / Voyage d'Italie, Paris, Tchou éditeur, 1967. 
VIAL, Charles-Éloi, « Police et censure du premier empire à la monarchie de juillet : la tumultueuse histoire de trois manuscrits du Marquis de Sade », Bibliothèque nationale de France, « Revue de la BNF » 2014/2 n 47, p. 58-67 (ISSN 1254-7700 ISBN 978271772597 Article disponible en ligne à l'adresse : https:// www.cairn.info/revue-de-la-bibliotheque-nationale-defrance-2014-2-page-58. htm, consulté le 05/03/2019).

WRIGHT, Doug, Quills and other plays, New York, Farrar, Strauss and Giroux, 2005.

\section{Markiz de Sade, Carstvo i carstvo metafora: quills i ostala pera}

Svjesno višeznačan naslov najavljuje analizu koja će se odvijati u dva dijela: najprije pokušati uspostaviti vezu između posljednjih godina života Markiza de Sadea (prema istoimenom svesku 25 korespondencije Markiza de Sadea, koja pokriva razdoblje od 1789. do 1814.), koji umire u Charentonu 2.12.1814., i razdoblja Carstva. U društvu obilježenom cenzurom, on paradoksalno, u umobolnici uspostavlja carstvo slobode strasti pod okriljem kazališta koje je napisano, stvoreno i uprizoreno.

Iz ovog odnosa između azila i teatra, koji podrazumijeva Foucaultovu »moć psihijatrije«, u ovom carstvu strasti rađa se izvjesna scena u Rancièreovom smislu ovoga pojma, scena suvremene metaforizacije.

Propadanju modela teatralnosti koja je specifična za libertinski roman ovdje odgovara interpretacija Sadea koju daje Ivo Brešan u svom romanu Prokletnici, kroz metaforu hrvatskog društva u tranziciji, i ona američka iz kazališnog komada Douga Wrighta, Quills i njegove metafore pisanja. Bilanca je neizvjesna.

Ključne riječi: Markiz de Sade, Empire, azil, teatar, scena, libertinski roman, Brešan, Wright 